\title{
BaRatin-SFD, analyse bayésienne des courbes de tarage à double échelle et de leurs incertitudes
}

\author{
Valentin MANSANAREZ ${ }^{1,2,3}$, Raphaël LE BOURSICAUD ${ }^{4}$ Jérôme LE COZ ${ }^{1}$, Benjamin RENARD', \\ Michel LANG ${ }^{1}$, Ivan HORNER ${ }^{1}$, Gilles PIERREFEU ${ }^{4}$, Karine POBANZ ${ }^{4}$
}

\author{
${ }^{I}$ Irstea, UR HHLY, Hydrologie-Hydraulique, centre de Lyon-Villeurbanne, 5 rue de la Doua, BP 32108, F-69616 Villeurbanne Cedex, France, \\ valentin.mansanarez@natgeo.su.se \\ ${ }^{2}$ Stockholm University, Department of Physical Geography, Stockholm, 106 91, Sweden \\ ${ }^{3}$ Bolin Centre for Climate Research, Stockholm University, Stockholm, 106 91, Sweden \\ ${ }^{4}$ Compagnie Nationale du Rhône (CNR), Centre d'Analyses Comportementales des Ouvrages Hydrauliques (CACOH), 4 rue de \\ Chalon-sur-Saône,69007Lyon, France, g.pierrefeu@cnr.tm.fr
}

\begin{abstract}
RÉSUMÉ. - Une méthode bayésienne à base hydraulique est introduite pour le développement des courbes de tarage hauteur-dénivelée-débit (SFD) utilisées pour les stations à double échelle et l'estimation de leurs incertitudes. Un contrôle par chenal avec une pente d'énergie variable due à un remous variable est considéré en combinaison avec un contrôle non influencé par le remous, habituellement un autre contrôle par chenal. La succession entre le contrôle influencé par le remous variable et le contrôle non influencé est résolue par continuité de la relation hauteur-débit. La différence entre les niveaux de référence des zéros des deux échelles limnimétriques est estimée comme un autre paramètre incertain du modèle SFD. On présente les résultats d'une station à double échelle typique d'une influence par le remous variable d'un barrage. L'exactitude et l'incertitude des débits estimés semblent acceptables, même lorsque l'incertitude sur la hauteur de transition entre les deux contrôles influencé et non influencé par le remous est élevée.
\end{abstract}

Mots-clés : Hydrométrie, débit, courbe de tarage, double échelle, remous variable, inférence bayésienne

\section{BaRatin-SFD, Bayesian analysis of rating curves at twin-gauge stations and their uncertainties}

\begin{abstract}
An original hydraulics-based Bayesian method is introduced for developing stage-fall-discharge (SFD) rating curves used at twin-gauge stations and estimating their uncertainties. A channel control with variable energy slope due to variable backwater is considered in combination with a backwater-unaffected control, usually another channel control. Succession between backwater-affected and backwater-unaffected controls is solved based on the continuity of the stage-discharge relation. The difference between the reference levels at the two stations is estimated as another uncertain parameter of the SFD model. Results at one typical twin-gauge station affected by the backwater of a run-of-the-river dam are presented. The accuracy and uncertainty of predicted discharges appear to be acceptable even when the uncertainty of the transition between backwater-affected and backwater-unaffected controls is high.
\end{abstract}

Key-words: Hydrometry, discharge, rating curve, twin-gauge, variable backwater, Bayesian inference

\section{INTRODUCTION}

La plupart des enregistrements de débit sont établis à partir du croisement du suivi du niveau d'eau au droit d'une station hydrométrique et d'une série de modèles hauteur-débit appelés " courbes de tarage », reliant le niveau mesuré à un débit. Ce mode de calcul n'est en fait valide que si la relation hauteur-débit est univoque, lorsqu'elle est régie soit par un contrôle par section (en amont d'une section critique) soit par un contrôle par chenal (écoulement dominé par les frottements) à pente d'énergie constante. La situation idéale est celle d'un écoulement uniforme lorsque le profil d'énergie est parallèle à la ligne d'eau et à la ligne du fond du lit. Dans certains cas, la pente d'énergie d'un contrôle par chenal peut être variable, en général en raison d'une condition limite variable en aval (« remous variable »), ou parfois à cause de conditions d'écoulement transitoire (« débit variable »). Des explications plus détaillées peuvent être trouvées dans Schmidt [2002] et Petersen-Øverleir et Reitan [2009].
L'importance de la gestion des stations hydrométriques affectées par une pente d'énergie variable a été reconnue depuis longtemps dans les manuels d'hydrométrie et dans les normes [Réméniéras, 1949, Rantz, 1982, Callède et al., 2001, ISO 9123:2001]. La pratique traditionnelle consiste à mesurer la dénivelée et donc la pente de la ligne d'eau entre l'échelle principale et une échelle secondaire située plus en aval ou très rarement en amont. Tant que les variations de la géométrie de l'écoulement et de la vitesse le long du tronçon restent limitées, on dit que le régime est " graduellement varié » et les formules usuelles de frottement (Darcy-Weisbach, Manning-Strickler, Chézy), faites pour le régime uniforme, peuvent encore être utilisées. Selon ces formules, le débit est proportionnel à la racine carrée de la pente d'énergie. C'est la base des calculs traditionnels de débit associés aux stations à double échelle (ISO 9123:2001). Pour autant, la relation hauteur-dénivelée-débit est le plus souvent disponible sous forme d'abaques (collection de courbes hauteur-débit pour différents niveaux aval), sans mention explicite des incertitudes associées. 
Pour apporter de nouvelles solutions à ce problème, ce travail s'appuie sur la piste de travail ouverte par Petersen-Øverleir et Reitan [2009]. L'approche bayésienne qu'ils ont introduite semble être la manière la plus prometteuse d'analyser les relations hauteur-dénivelée-débit tout en quantifiant les incertitudes associées à ces relations. Plus généralement, les méthodes bayésiennes fournissent un cadre flexible pour incorporer des informations préalables sur la segmentation et l'extrapolation du modèle au-delà des débits jaugés les plus hauts. Elles peuvent prendre en compte les incertitudes individuelles des jaugeages et fournir des résultats d'incertitude complets sous la forme de distributions probabilistes [Moyeed et Clarke, 2005]. Plus important encore, elles permettent la décomposition des composantes d'incertitude liées au modèle de la courbe de tarage («erreur structurelle»), à l'estimation des paramètres («erreur paramétrique») et potentiellement les erreurs systématiques et non systématiques des séries de hauteur d'eau («erreur de propagation»).

\section{MÉTHODE}

\section{II.1. Analyse bayésienne des courbes de tarage}

La méthode proposée pour évaluer les courbes de tarage à double échelle a été implémentée dans le cadre bayésien associé à la méthode BaRatin [Le Coz et al., 2013, Le Coz et al., 2014]. BaRatin permet de construire des courbes de tarage hauteur-débit avec l'estimation des incertitudes associées, en combinant à la fois la connaissance a priori sur les contrôles hydrauliques et l'information des jaugeages incertains (voir Figure 1 et [Le Coz et al., 2014]). Le modèle de la courbe de tarage est obtenu à partir de la combinaison de fonctions puissance correspondant à chaque contrôle hydraulique connu ou supposé (étape 1 sur la Figure 1). L'utilisateur définit également les distributions a priori des paramètres physiques de ce modèle hauteur-débit, « a priori » signifiant sans regarder les jaugeages utilisés dans l'inférence bayésienne (étape 2 sur la Figure 1). Cette inférence permet d'obtenir une distribution cible, nommée distribution a posteriori qui, grâce au théorème de Bayes, incorpore à la fois l'information a priori sur ces paramètres physiques mais aussi l'information contenue dans les jaugeages à travers la fonction de vraisemblance. La distribution a posteriori est ensuite échantillonnée en utilisant les techniques de Monte-Carlo par Chaînes de Markov (MCMC), ce qui permet d'obtenir un ensemble d'échantillons des paramètres de la courbe de tarage, c'est-à-dire un ensemble de courbes de tarage (étape 3 sur la Figure 1). Tout conflit physique entre les résultats et les a priori supposés doit être vérifié et doit conduire à remettre en question soit le modèle de la courbe de tarage soit les incertitudes estimées des jaugeages. Un post-traitement statistique de cet ensemble de courbes de tarage, ou «spaghetti», permet d'obtenir les intervalles d'incertitude de la courbe de tarage et des séries temporelles de débit à n'importe quel niveau de probabilité (étape 3 sur la Figure 1). La courbe de tarage de l'estimateur du maximum a posteriori (courbe MAP) est calculée en utilisant l'échantillon de paramètres maximisant la distribution a posteriori.

La méthode BaRatin a été adaptée au cas des courbes de tarage à double échelle.

La hauteur $h$ a été remplacée par deux hauteurs $h_{1}$ et $h_{2}$ mesurées respectivement aux échelles principale et secondaire. Les jaugeages $\left(\tilde{h}_{1, i}, \tilde{h}_{2, i}, \tilde{Q}_{i}\right)_{i=1 N}$ sont vus comme des estimations des valeurs réelles $\left(h_{1, i}, h_{2, i}, Q_{i}\right)_{i=1, N}$ des hauteurs d'eau et du débit en supposant que les erreurs de mesure sur la hauteur d'eau sont négligeables devant les erreurs de mesure $\varepsilon_{Q, i}$ du débit, supposées suivre une loi gaussienne de moyenne 0 et d'écart-type $u_{Q, i}$ :

$$
\left\{\begin{array}{c}
\tilde{h}_{1, i}=h_{1, i} \\
\tilde{h}_{2, i}=h_{2, i} \\
\tilde{Q}_{i}=Q_{i}+\varepsilon_{Q, i} \operatorname{avec} \varepsilon_{Q, i} \sim N\left(0, u_{Q, i}\right)
\end{array}\right.
$$

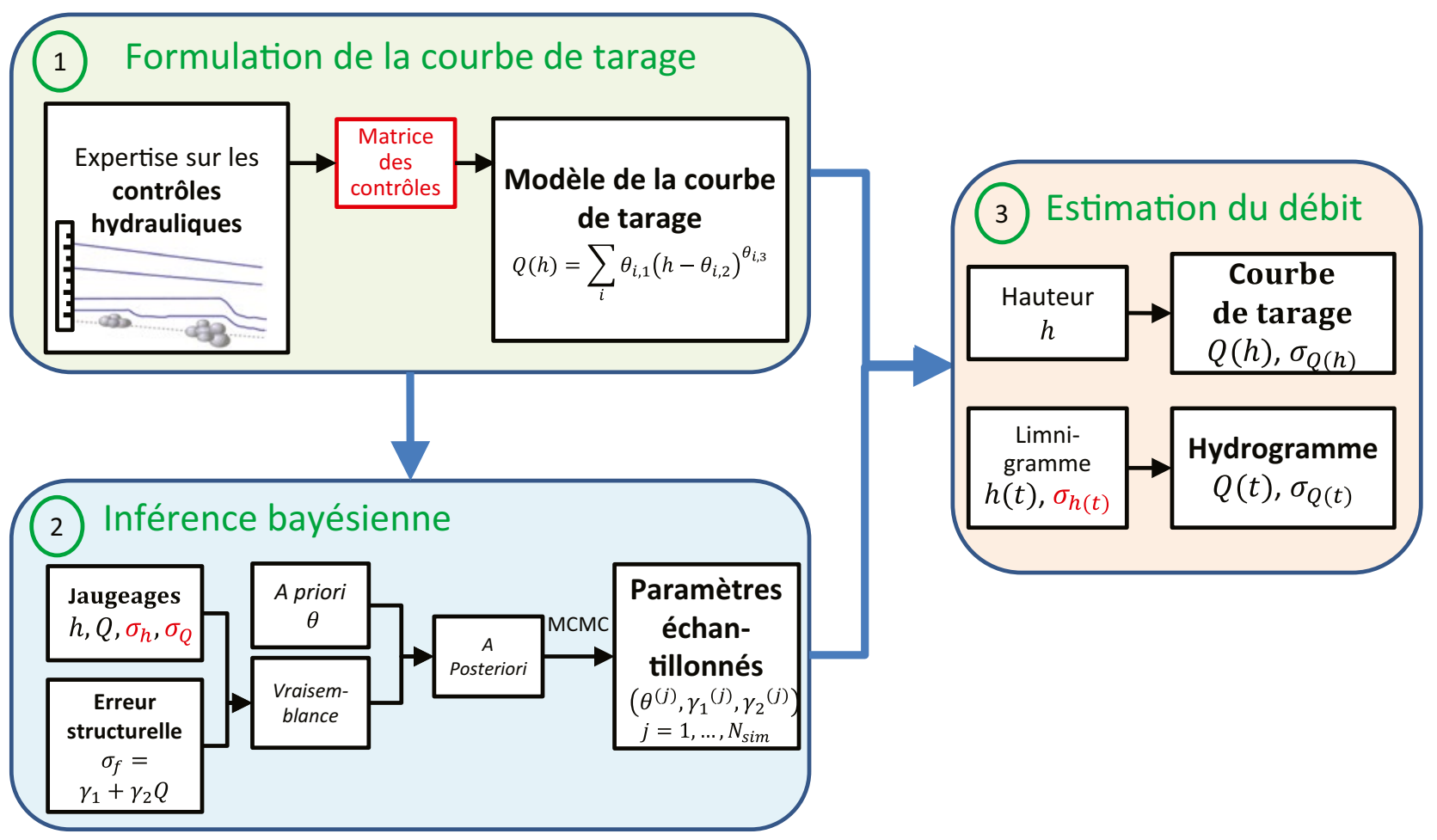

Figure 1 : Principes de la méthode BaRatin pour l'estimation des courbes de tarage hauteur-débit et de leurs incertitudes 
D'autre part, la courbe de tarage $\widehat{Q}=f\left(h_{1}, h_{2} \mid \theta\right)$ est considérée comme une approximation de la vraie relation hauteur-débit, et est donc entachée d'une erreur structurelle $\varepsilon_{f}$ modélisée comme suit :

$$
\begin{gathered}
Q_{i}=f\left(h_{i 1,}, h_{2, i} \mid \theta\right)+\varepsilon_{f, i} \\
\operatorname{avec} \varepsilon_{f, i} \sim N\left(0 ; \sigma_{f, i}=\gamma_{1}+\gamma_{2} \widehat{Q}_{i}\right)
\end{gathered}
$$

où $\theta=\left(\theta_{1}, \ldots, \theta_{N_{p a r}}\right)$ sont les paramètres de la courbe de tarage. Le modèle d'erreur affine $\sigma_{f}=\gamma_{1}+\gamma_{2} \widehat{Q}$ suppose que l'incertitude structurelle $\sigma_{f}$ de la courbe de tarage est dominée par un terme $\gamma_{1}$ constant à bas débit et par un terme $\gamma_{2}$ proportionnel au débit estimé $\widehat{Q}$ à haut débit. Ces deux paramètres d'erreur sont estimés pour refléter toute l'incompatibilité entre les observations (jaugeages) et le modèle (courbe de tarage hauteur-dénivelée-débit) qui ne peut être expliquée par l'incertitude des jaugeages.

La combinaison des équations (1) et (2) permet d'obtenir la relation hauteur-dénivelée-débit suivante entre les valeurs observées (en supposant que les erreurs de mesure et les erreurs structurelles sont indépendantes) :

$$
\begin{gathered}
\tilde{Q}_{i}=f\left(\tilde{h}_{i 1}, \tilde{h}_{2, i} \mid \theta\right)+\varepsilon_{Q, i}+\varepsilon_{f, i} \\
\operatorname{avec}\left(\varepsilon_{Q, i}+\varepsilon_{f, i}\right) \sim N\left(0 ; \sqrt{u_{Q, i}^{2}+\sigma_{f, i}^{2}}\right)
\end{gathered}
$$

Les quantités inconnues dans l'équation (3) qui doivent être estimées par l'inférence bayésienne sont les paramètres $\theta$ de la courbe de tarage et les paramètres $\gamma_{1}$ et $\gamma_{2}$ du modèle d'erreur structurelle. Dans les sections qui suivent, les intervalles d'incertitude sont calculés à partir des échantillons MCMC à un niveau de probabilité de $95 \%$ (c'est-à-dire les quantiles $2,5 \%$ et $97,5 \%$ ), comme considéré habituellement dans les applications hydrométriques (ISO/TS 25377:2007, ISO 9123:2001).

\section{II.2. Le modèle hauteur-dénivelée-débit (SFD)}

Nous introduisons un modèle basé sur des hypothèses hydrauliques simples pour les régimes d'écoulement graduellement varié et uniforme dans les chenaux larges avec une condition de transition simple entre les deux contrôles influencé et non influencé :

$Q\left(h_{1}, h_{2}\right)= \begin{cases}K_{S} B\left(h_{1}-h_{0}\right)^{M} \sqrt{\left(h_{1}-h_{2}-\delta_{h}\right) / L} & \text { si } h_{1}<\kappa\left(h_{2}\right)(\text { pentevariable) } \\ K_{S}^{\prime} B^{\prime}\left(h_{1}-h_{0}^{\prime}\right)^{M^{\prime}} \sqrt{S_{0}} & \text { si } h_{1} \geq \kappa\left(h_{2}\right)(\text { penteconstante) }\end{cases}$

où $Q$ est le débit, $h_{1}$ la hauteur d'eau à l'échelle principale, $h_{2}$ la hauteur d'eau à l'échelle secondaire, $L$ la distance

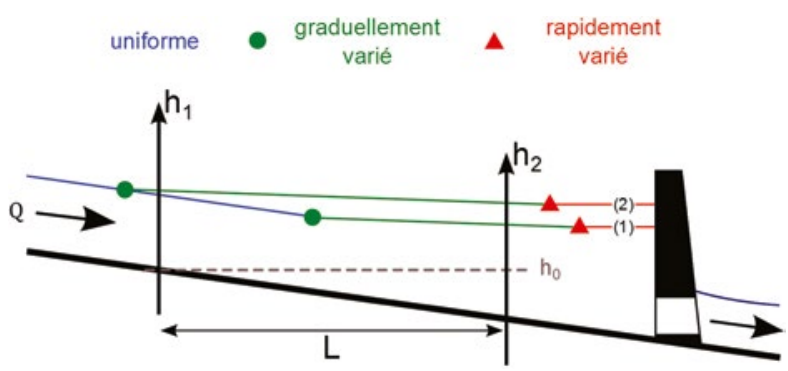

Figure 2 : Modèle hauteur-dénivelée-débit (SFD), transition typique entre un contrôle par chenal sous influence aval variable d'un barrage (ligne d'eau 2) et un contrôle par chenal uniforme, non influencé par le barrage (ligne d'eau 1) entre les deux échelles et $\delta_{h}$ la différence de référence altimétrique entre les zéros des deux échelles. Les paramètres du contrôle par chenal influencé (entre les deux échelles) sont les suivants : $K_{S}$ est le coefficient de Strickler ; $B^{\prime}$ est la largeur moyenne de la rivière ; $h_{0}$ est la cote moyenne $\mathrm{du}$ fond du lit de la rivière (offset) et $M$ est l'exposant. Les paramètres du contrôle par chenal non influencé (autour de l'échelle principale) : $K_{S}$ est le coefficient de Strickler ; $B^{\prime}$ est la largeur moyenne de la rivière ; $M^{\prime}$ est l'exposant ; $h_{0}$ est la cote moyenne du fond du lit de la rivière (offset) et $S_{0}$ est la pente du fond de la rivière.

L'équation (4) est divisée en deux parties : la première (pente variable) correspond au contrôle par chenal sous influence aval variable avec une pente d'énergie variable alors que la seconde partie (pente constante) correspond au contrôle par chenal non influencé. Les deux équations sont dérivées de l'équation de Manning-Strickler en supposant une section transversale rectangulaire avec un grand rapport largeur / profondeur ( $M$ et $M^{\prime}$ devraient donc être proches de 5/3).

La transition entre les deux contrôles est considérée comme un simple point d'intersection (voir Figure 2) : le régime d'écoulement devient uniforme lorsque la hauteur $h_{1}$ de l'échelle principale est supérieure à $\kappa\left(h_{2}\right)$, une certaine hauteur de transition qui dépend de la valeur de la hauteur d'eau $h_{2}$ à l'échelle secondaire. Une différence majeure avec le modèle SFD proposé par Petersen-Øverleir et Reitan (2009) est que la condition de transition est une condition de continuité du débit de la courbe de tarage $Q\left(h_{1}, h_{2}\right)$ en $h_{1}=\kappa\left(h_{2}\right)$. La hauteur de transition $\kappa\left(h_{2}\right)$ est calculée par résolution numérique (Newton-Raphson) appliquée à la fonction $\Phi$ suivante :

$\Phi(x)=K_{S} B\left(x-h_{0}\right)^{M} \sqrt{\left(x-h_{2}-\delta_{h}\right) / L}-K_{S}^{\prime} B^{\prime}\left(x-h_{0}^{\prime}\right)^{M^{\prime}} \sqrt{S_{0}}(5)$

Puisqu'il s'agit d'une source d'erreur indépendante et potentiellement importante, la différence $\delta_{h}$ de référence entre les zéros des deux échelles est estimée comme un paramètre.

Ce modèle SFD a été développé par Mansanarez [2016] au cours de sa thèse. Des explications plus détaillées peuvent être trouvées dans Le Coz et al. [2016b] et Mansanarez et al. [2016].

Dans la section suivante, ce modèle est appliqué à une station à double échelle typiques exploitées par la Compagnie nationale du Rhône (CNR) sur un site de contrôle par chenal, ce qui correspond à la situation la plus courante. Plusieurs stations similaires sont situées sur les affluents du Rhône, en France, dans leur partie la plus basse affectée par le remous variable des centrales hydroélectriques construites dans le cours principal du Rhône. La quantification et la réduction des incertitudes des débits calculés à ces stations, en particulier les bas débits, revêtent une importance opérationnelle forte pour optimiser la production d'énergie et respecter les débits réglementaires.

\section{APPLICATION : L'ISÈRE À BEAUMONT-MONTEUX, FRANCE}

\section{III.1. Présentation de la station hydrométrique}

Avec un bassin versant de $11890 \mathrm{~km}^{2}$ pour un débit moyen interannuel de $333 \mathrm{~m}^{3} \cdot \mathrm{s}^{-1}$, l'Isère est le second affluent du Rhône en terme de débit moyen. Les mesures de débit sont réalisées par la CNR sur l'Isère entre l'aménagement hydroélectrique de Beaumont-Monteux (échelle principale : 
4501'01.1'N, 4'54'49.1'E) et la confluence avec le Rhône (échelle secondaire : 4459'41.2”N, 452’12.4”E). Ce tronçon de contrôle est influencé par le remous variable de l'aménagement hydroélectrique de Bourg-lès-Valence située plus loin en aval sur le Rhône (à environ $4,75 \mathrm{~km}$ de l'échelle principale). La hauteur d'eau à ce barrage est ajustée selon les règles d'exploitation pour les débits habituels. Quand le débit dans le Rhône et l'Isère dépasse un certain seuil, les vannes sont ouvertes graduellement et l'effet d'influence aval disparait pour les crues les plus importantes [Dugué et al., 2015].

La configuration hydraulique de la station a été modélisée en utilisant le modèle SFD (équation 4) pour deux contrôles par chenal, l'un influencé et l'autre non influencé par le remous variable. Pour chaque paramètre du modèle SFD, on a déterminé des valeurs a priori (voir tableau 1) en utilisant des cartes et des images satellites, un modèle hydrodynamique 1D existant [Dugué et al., 2015], des profils en travers disponibles (y compris des transects ADCP), et les informations sur le nivellement des deux échelles. La largeur de la rivière augmente graduellement de l'échelle principale à l'échelle secondaire, ce qui se reflète dans l'incertitude de $B$ et $B^{\prime}$. Malgré des données géométriques précises, la distance $L$ entre les deux échelles est laissée légèrement incertaine pour tenir compte de la sinuosité de l'Isère. La pente $S_{0}$ de la ligne d'eau hors influence aval a été estimée à partir des enregistrements de hauteur d'eau, en crue, entre les deux échelles.

$\mathrm{Au}$ total, 46 jaugeages ont été réalisés entre $128 \mathrm{~m}^{3} \cdot \mathrm{s}^{-1}$ et $1389 \mathrm{~m}^{3} \cdot \mathrm{s}^{-1}$, ce qui couvre à la fois les situations d'influence aval par le remous variable et les situations sans influence. Les hauteurs d'eau $h_{1}$, observées à l'échelle principale, varient entre 0,81 et $3,47 \mathrm{~m}$ tandis que celles $h_{2}$ observées à l'échelle secondaire varient entre 0,20 et $0,75 \mathrm{~m}$. Quelques-uns des jaugeages ont été réalisés à la suite, ce qui peut violer l'hypothèse d'erreurs indépendantes entre les jaugeages. La plupart des jaugeages ont été réalisés avec un ADCP sur un bateau mobile dans des conditions de mesure favorables. Les incertitudes ont donc été fixées à $5 \%$ (niveau de confiance de $95 \%$ ) pour toutes les mesures par ADCP. Les incertitudes des jaugeages réalisés par courantomètre suspendus par câble ont été fixées à $7 \%$. Ces valeurs d'incertitude proviennent du jugement d'experts et des résultats d'expériences interlaboratoires lorsqu'elles sont disponibles [Le Coz et al., 2016a].

\section{III.2. Estimation des courbes de tarage}

Les résultats a posteriori sont en accord avec les a priori (voir Tableau 1 et Figure 3). La valeur absolue de la différence $\delta_{h}$ de référence altimétrique entre les zéros des deux échelles est légèrement supérieure à ce que l'on pourrait attendre de la précision du nivellement. Cette valeur calée peut aussi compenser l'erreur de non-linéarité de la ligne d'eau entre les deux échelles, notamment. Le coefficient $K_{S} B$ et l'offset $h_{0}$ du contrôle par chenal influencé par le remous variable semblent être fortement corrélés entre eux (voir Figure 3), ainsi que les trois paramètres, $K_{S} B S_{0}, h_{0}$ et $M^{\prime}$, du contrôle non influencé. Les estimations des composantes de l'incertitude structurelle $\gamma_{1}$ et $\gamma_{2}$ (voir Tableau 1) sont faibles, respectivement de l'ordre de quelques $\mathrm{m}^{3} \cdot \mathrm{s}^{-1}$ et de $1 \%$ à $2 \%$. De tels résultats suggèrent que le modèle SFD est assez adéquat pour représenter la relation hauteur-débit sur ce site.

Le modèle SFD donne les formes attendues des trajectoires hauteur-débit (voir figure 4), avec un faisceau continu (fonction de $h_{2}$ ) de courbes influencées par le remous variable qui fusionnent doucement avec la courbe non influencée par des transitions distinctes dépendant de la valeur de la hauteur $h_{2}$ à l'échelle secondaire. L'accord entre les deux types de courbes et l'ensemble des jaugeages est satisfaisant quelle que soit la valeur de $h_{2}$ : les différences entre les débits mesurés et les débits estimés par le modèle BaRatin-SFD (courbe MAP) sont inférieures à $\pm 5 \%$ et les intervalles d'incertitude à $95 \%$ des estimations de débits sont également de $\pm 5 \%$ environ, sauf pour les débits inférieurs à $200 \mathrm{~m}^{3} \cdot \mathrm{s}^{-1}$ (environ $\pm 10 \%$ ). Néanmoins, les intervalles d'incertitude sont peut-être sous-estimés car les erreurs corrélées entre les jaugeages successifs n'ont pas été prises en compte dans les estimations.

Tableau 1: Paramètres a priori et a posteriori du modèle SFD de l'Isère à Beaumont-Monteux. $N(\mu ; \sigma)$ désigne la distribution gaussienne de moyenne $\mu$ et d'écart-type $\sigma$, tandis que $U(a ; b)$ désigne la distribution uniforme de bornes a et $b$.

\begin{tabular}{|c|c|c|c|c|}
\hline \multirow{2}{*}{$\begin{array}{l}\text { Paramètres } \\
\text { physiques }\end{array}$} & \multirow{2}{*}{ Unités } & \multirow{2}{*}{$\begin{array}{l}\text { Distributions } \\
\text { a priori }\end{array}$} & \multicolumn{2}{|c|}{ Résultat a posteriori } \\
\hline & & & MAP & Quantiles $2.5 \%$ et $97.5 \%$ \\
\hline$K_{S} B$ & {$\left[\mathrm{~m}^{4 / 3} \cdot \mathrm{s}^{-1}\right]$} & $\mathrm{N}(4500 ; 710)$ & 4834 & {$[4072,5834]$} \\
\hline $\boldsymbol{h}_{0}$ & {$[\mathrm{~m}]$} & $\mathrm{N}(-1 ; 0.5)$ & -1.45 & {$[-1.74,1.14]$} \\
\hline$M$ & {$[-]$} & $\mathrm{N}(1.667 ; 0.025)$ & 1.670 & {$[1.622,1.714]$} \\
\hline$L$ & {$[\mathrm{~m}]$} & $\mathrm{N}(4750 ; 100)$ & 4749 & {$[4562,4947]$} \\
\hline $\boldsymbol{h}_{0}^{\prime}$ & {$[\mathrm{m}]$} & $\mathrm{N}(-1 ; 2)$ & -0.59 & {$[-0.68,-0.50]$} \\
\hline$K_{S}^{\prime} B^{\prime} \sqrt{S_{0}}$ & {$\left[\mathrm{~m}^{4 / 3} \cdot \mathrm{s}^{-1}\right]$} & $\mathrm{N}(120 ; 20)$ & 127.8 & {$[116.4,140.8]$} \\
\hline$\delta_{h}$ & {$[\mathrm{~m}]$} & $\mathrm{N}(0 ; 0.1)$ & -0.106 & {$[-0.158,-0.072]$} \\
\hline$M^{\prime}$ & {$[-]$} & $\mathrm{N}(1.667 ; 0.025)$ & 1.679 & {$[1.629,1.722]$} \\
\hline$\gamma_{1}$ & {$\left[\mathrm{~m}^{3} \cdot \mathrm{s}^{-1}\right]$} & $\mathrm{U}\left(0 ; 10^{6}\right)$ & 0.39 & {$[0.04,5.53]$} \\
\hline$\gamma_{2}$ & {$[-]$} & $\mathrm{U}\left(0 ; 10^{6}\right)$ & 0.0087 & {$[0.0004,0.0231]$} \\
\hline
\end{tabular}




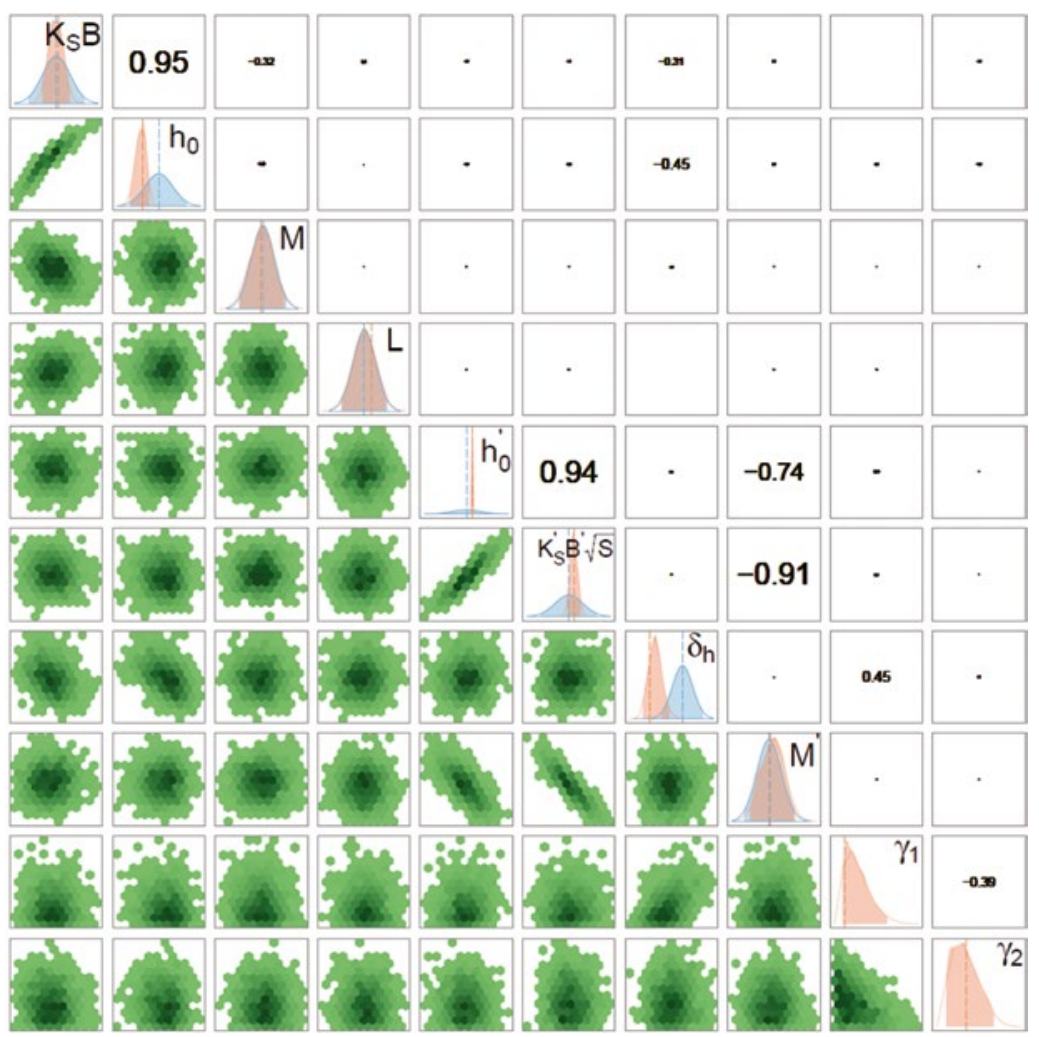

Figure 3 : BaRatin-SFD appliqué à l'Isère à Beaumont-Monteux, résultats des échantillonnages MCMC. La diagonale correspond aux densités marginales a priori (bleu) et a posteriori (rouge). Le triangle supérieur de la matrice correspond à la matrice de corrélation des paramètres tandis que le triangle inférieur présente les nuages de points des valeurs échantillonnées.

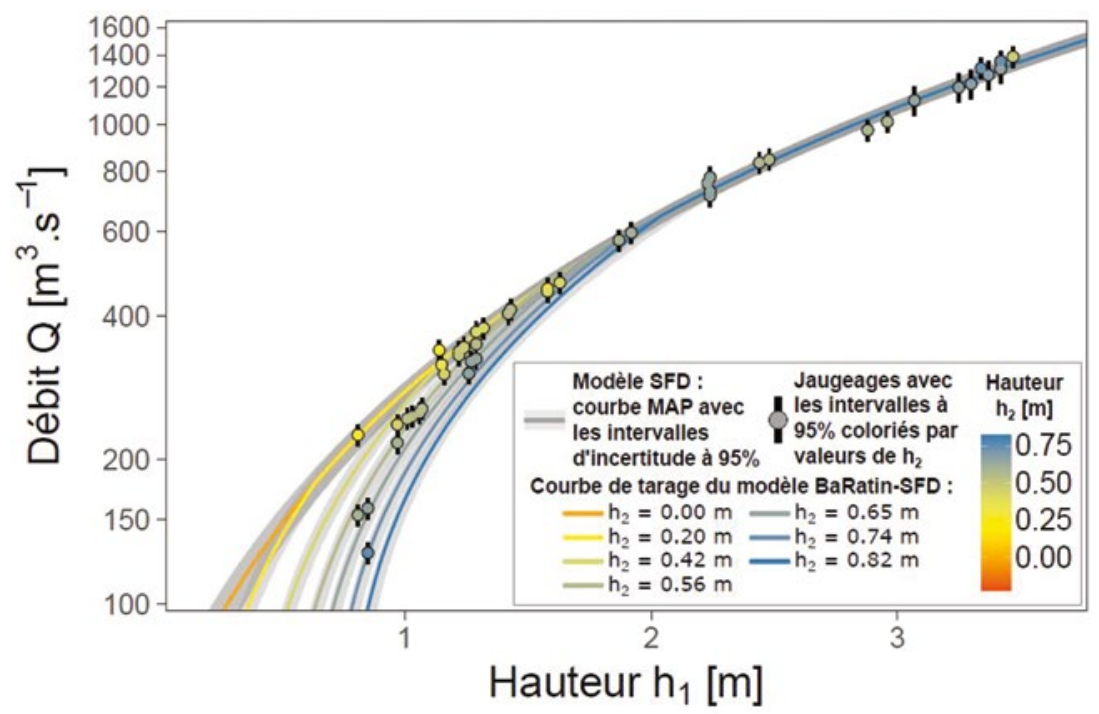

Figure 4 : L'Isère à Beaumont-Monteux : représentation hauteur-débit des résultats a posteriori du modèle hauteur-dénivelée-débit (SFD), avec les débits en échelle logarithmique. La courbe de tarage SFD est tracée seulement pour 7 valeurs de $h_{2}$ qui correspondent à des valeurs de jaugeages $\left(h_{2}=0.20,0.42,0.56,0.65\right.$ et $\left.0.74 \mathrm{~m}\right)$ et aux valeurs extrêmes observées $(0.00 \mathrm{~m}$ et $0.82 \mathrm{~m})$.

\section{III.3. Estimation des séries de débit}

La Figure 5 représente les débits estimés $Q\left(h_{1}, h_{2}\right)$ par le modèle BaRatin-SFD pour la fin d'avril 2015, ainsi que le débit de transition $Q\left(\kappa\left(h_{2}\right), h_{2}\right)$ entre les deux situations : sous influence du remous variable (en dessous du débit de transition) ou non influencé (au-dessus). Au cours de cette semaine, présentant des pics quotidiens et une crue typiques de ce site, le contrôle actif est soit influencé par le remous variable, soit non influencé selon la valeur du débit et de la hauteur d'eau $h_{2}$ de l'échelle secondaire. La transition prédite est hydrauliquement compatible avec les règles de fonctionnement de l'aménagement hydroélectrique de Bourg-lès-Valence, qui est progressivement abaissé lorsque des crues se produisent pour atténuer et finalement annuler les effets du remous variable.

En dépit des incertitudes variables et non négligeables du débit de transition, les incertitudes des débits prédits demeurent beaucoup plus faibles et acceptables (en dessous 


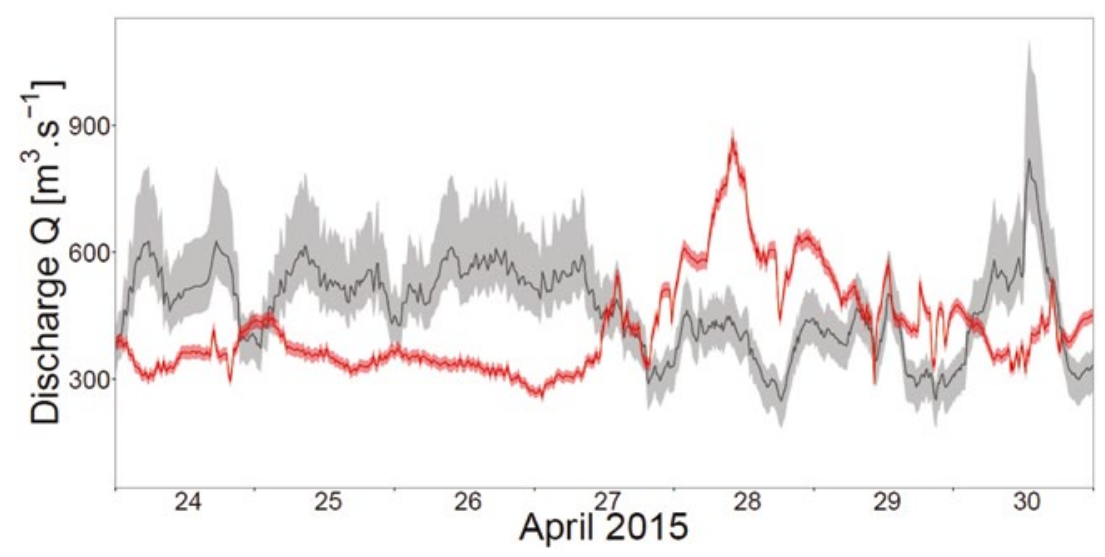

Figure 5 : L'Isère à Beaumont-Monteux, fin du mois d'avril 2015, représentation temporelle des débits estimés par BaRatin-SFD (courbe MAP et intervalles d'incertitude à $95 \%$ ). En rouge, sont représentés les débits estimés $Q\left(h_{1}, h_{2}\right)$ alors qu'en gris, les débits de transition $Q\left(\kappa\left(h_{2}\right), h_{2}\right)$ correspondent à la hauteur de transition estimée entre le contrôle par chenal sous influence aval du remous variable et le contrôle par chenal non-influencé.

de $\pm 10 \%$ ). Cela peut être dû au fait que les équations des deux contrôles sont similaires au voisinage de la transition, où elles fusionnent en douceur (voir Figure 4).

\section{CONCLUSIONS}

Une méthode bayésienne originale à base hydraulique a été mise en place pour l'élaboration des courbes de tarage hauteur-dénivelée-débit (SFD) utilisées pour les stations à double échelle et l'estimation de leurs incertitudes. Un modèle simple a été proposé pour tenir compte d'un contrôle par chenal influencé par un remous variable combiné à un contrôle par chenal non influencé par le remous. Notez que toute équation de courbe de tarage non influencée par le remous peut être utilisée, y compris celle correspondant à un contrôle par section, le cas échéant. La transition entre le contrôle influencé et les contrôles non influencés est résolue par une simple condition de continuité sur les débits. Le modèle SFD a été mis en œuvre dans le cadre actuel de BaRatin.

La performance de la nouvelle méthode a été illustrée à l'aide d'un cas d'application typique affecté par le remous variable d'un barrage de rivière : l'Isère à Beaumont-Monteux, en France. Le domaine hauteur-dénivelée-débit a été documenté par des jaugeages réalisés sur une gamme d'écoulement influencé ou non par le remous variable. L'exactitude et l'incertitude des débits estimés ont été jugées satisfaisantes, même lorsque la transition entre les deux contrôles était plus incertaine. Les courbes de tarage SFD obtenues sont en bon accord avec les observations (jaugeages) et leurs enveloppes d'incertitude sont acceptables pour le calcul des chroniques de débit. L'incertitude structurelle a été estimée faible par rapport à l'incertitude paramétrique, ce qui suggère que le modèle de courbe de tarage proposé représente ce type d'écoulement assez correctement. Cependant, la sensibilité des résultats aux erreurs éventuellement corrélées dans les jaugeages réalisés successivement avec la même instrumentation et la même procédure de mesure devrait être testée. Des conclusions similaires ont été tirées de l'application de la méthode à d'autres sites.

La méthode proposée présente les avantages d'être basée sur des équations hydrauliques simples et de fournir des résultats de courbes de tarage, tant sous forme d'équations que de barèmes (tableaux). Les résultats d'incertitude viennent avec, sous forme de distributions probabilistes échantillonnées à partir desquelles toute analyse statistique peut être effectuée. L'analyse de l'incertitude peut en outre intégrer la propagation des incertitudes de mesure des hauteurs d'eau aux deux échelles. Les résultats préliminaires de la propagation confirment que ces incertitudes sur les hauteurs peuvent détériorer la qualité des estimations des séries de débits lorsque la dénivelée est faible entre les deux échelles.

\section{REMERCIEMENTS}

Le développement de la méthode BaRatin depuis 2010 est soutenu par Irstea, le SCHAPI (services hydrologiques nationaux français), et la Compagnie nationale du Rhône (CNR). Le modèle BaRatin-SFD [Mansanarez et al., 2016] pour les stations à double échelle a été mis au point par V. Mansanarez, dont la thèse [Mansanarez, 2016] a été co-financée par Irstea et la CNR, avec le soutien du SCHAPI. La méthode a bénéficié des discussions avec de nombreux hydromètres et hydrologues de services opérationnels de plusieurs pays, notamment Philippe Vauchel (IRD). BaRatin utilise la bibliothèque Fortran DMSL développée par Dmitri Kavetski de l'Université d'Adélaïde, en Australie.

\section{RÉFÉRENCES}

Callède J., P. Kosuth, \& E. De Oliveira (2001) - Etablissement de la relation hauteur-débit de l'Amazone à Óbidos : Méthode de la dénivelée normale à " géométrie variable ». Hydrological Sciences Journal, 46(3), 451-463.

Dugue V., C. Walter E. Andries A. Launay J. Le Coz B. CAmenen J.-B. FAure (2015) - Accounting for hydropower schemes' operation rules in the 1D hydrodynamic modeling of the Rhône River from Lake Geneva to the Mediterranean Sea. E-proceedings of the 36th IAHR World Congress, 28 June-3 July, 2015, The Hague, the Netherlands, 8 p.

ISO (2007) - Iso/Ts 25377:2007. Hydrometric Uncertainty Guidance (Hug), $51 \mathrm{p}$.

ISO (2001) - Iso 9123:2001. Measurement Of Liquid Flow In Open Channels - Stage-Fall-Discharge Relationships, 14 p. 
Le Coz J., Chaleon C., Bonnifait L., Branger B., Le Boursicaud R., Renard B. Branger F., Diribarne J., VAlente M. (2013) - Analyse bayésienne des courbes de tarage et de leurs incertitudes : la méthode BaRatin. La Houille Blanche, 6, 31-41.

Le Coz J., Renard B., Bonnifait L., Branger F., Le Boursicaud R. (2014) - Combining hydraulic knowledge and uncertain gaugings in the estimation of hydrometric rating curves: a Bayesian approach. Journal of Hydrology, 509, 573-587.

Le Coz J., Blanquart B., Pobanz K., Dramais G., Pierrefeu G., Hauet A., AND Despax A. (2016) - Estimating the uncertainty of streamgauging techniques using In situ collaborative interlaboratory experiments. J. Hydraul. Eng, 10.1061/(ASCE) HY.1943-7900.0001109, 04016011.

Le Coz J., Mansanarez V., Renard B., Lang M., Pierrefeu G., Pobanz K., Le Boursicaud R. (2016) - Bayesian analysis of rating curves at twin gauge stations. IAHR River Flow conference, St. Louis, Missouri, USA, 12-15 July 2016, 7 p.

Mansanarez V. (2016) - Non unique stage-discharge relations: Bayesian analysis of complex rating curves and their uncertainties. Thèse de doctorat, Université Grenoble Alpes, Irstea Lyon-Villeurbanne, 240p.
Mansanarez V., Le Coz J., Renard B., Vauchel P., Pierrefeu G., LANG M. (2016) - Bayesian analysis of stage-fall-discharge rating curves and their uncertainties. Water Resources Research, 52, 7424-7443.

Moyeed R. ET R. T. Clarke (2005) - The use of Bayesian methods for fitting rating curves, with case studies. Advances in Water Resources, 28, 807-818.

Petersen-Øverleir A. et T. Reitan (2009) - Bayesian analysis of stage-fall-discharge models for gauging stations affected by variable backwater. Hydrological Processes, 23(21), 3057-3074.

RANTZ S. (1982) - Computation of discharge. Measurement and computation of streamflow, U. S. Geological Survey, Washington Water-Supply Paper, 2, 2175.

RÉMÉNIÉRAs G. (1949) - L'Hydraulique des stations limnimétriques pour la mesure du débit des cours d'eau [Hydrological annual report of France, year 1949: the Hydraulics of gauging stations for streamflow measurements (in French)]. Annuaire hydrologique de la France, année 1949: Société hydrotechnique de France.

SCHMidT A. (2002) - Analysis of stage-discharge relations for open-channel flows and their associated uncertainties. Thèse de doctorat, University of Illinois, Urbana-Champaign, 329p. 